\title{
Fresh versus frozen single embryo transfer in Chinese women of advanced age undergoing IVF/ICSI: a study protocol for a randomized controlled trial
}

\section{Miaoxin Chen}

Tongji University School of Medicine

\section{Yuanyuan Wu}

Tongji University School of Medicine

\section{Xin Huang}

Tongji University School of Medicine

Wenqiang Liu

Tongji University School of Medicine

\section{Wentao Li}

Monash University

Chunyan Sun

Tongji University School of Medicine

\section{Zhenzhen Meng}

Tongji University School of Medicine

\section{Ben W Mol}

\section{Monash University}

Xiaoming Teng ( $\nabla$ tengxiaoming@hotmail.com )

Tongji University School of Medicine https://orcid.org/0000-0001-6625-209X

\section{Study protocol}

Keywords: Frozen embryo transfer, advanced age, in vitro fertilization (IVF), intracytoplasmic sperm injection (ICSI), live birth, randomized controlled trial

Posted Date: March 24th, 2020

DOl: https://doi.org/10.21203/rs.3.rs-18972/v1

License: (c) (i) This work is licensed under a Creative Commons Attribution 4.0 International License. Read Full License 

1 Fresh versus frozen single embryo transfer in Chinese women of advanced age

2 undergoing IVF/ICSI: a study protocol for a randomized controlled trial

4 Miaoxin Chen ${ }^{1 *}$, Yuanyuan $\mathrm{Wu}^{1 *}$, Xin Huang ${ }^{1 *}$, Wen-Qiang Liu ${ }^{1 *}$, Wen-Tao $\mathrm{Li}^{2 *}$, Chunyan

5 Sun $^{1 *}$, Zhenzhen Meng ${ }^{1 *}$, Ben.W.Mol${ }^{2}$, Xiaoming Teng ${ }^{1}$

$6 \quad{ }^{*}$ These authors contributed equally to this article.

7

$8 \quad{ }^{1}$ Center for Reproductive Medicine, Shanghai First Maternity and Infant Hospital, Tongji

9 University School of Medicine, Shanghai, China

102 Department of Obstetrics and Gynaecology, Monash Medical Centre, Monash

11 University, Melbourne, Victoria, Australia

12

13 Corresponding author: Dr. Xiaoming Teng

14 Centre for Reproductive Medicine, Shanghai First Maternity and Infant Hospital, Tongji

15 University School of Medicine.

162699 Gaoke West Road, Pudong District, Shanghai, China, 201204

17 Email: tengxiaoming@hotmail.com

18 Phone: +86 2120261426

19 Fox: +862165982875

20

21

22 
Abstract

Background: Elective frozen cleavage embryo transfer resulted in significantly higher live birth rate in patients with polycystic ovary syndrome but not in ovulatory women compared with fresh embryo transfer. Further, elective single frozen blastocyst transfer had significantly higher live birth rate in ovulatory women compared with single fresh blastocyst transfer. However, it is unknown whether single frozen cleavage embryo

29 transfer results in higher cumulative live birth rate in women of advanced age 30 undergoing in vitro fertilization (IVF) or intracytoplasmic sperm injection (ICSI). The aim

31 of this study is to compare the effectiveness of frozen cleavage embryo transfer with 32 fresh cleavage embryo transfer in Chinese women of advanced age.

33 Methods: This study is a double-blind randomized controlled clinical trial (1:1 treatment 34 ratio of frozen embryo transfer vs. fresh embryo transfer). A total of 840 women of 35 advanced age with normal ovarian reserve undergoing the first cycle of IVF or ICSI will 36 be enrolled and randomized into two parallel groups. Participants in group A will 37 undergo frozen single cleavage embryo transfer, and participants in group B will 38 undergo fresh single cleavage embryo transfer. The primary outcome is the cumulative 39 live birth rate of the trial IVF/ICSI cycle within 12 months after randomization. This study 40 is powered to detect an absolute difference of $8 \%(23 \%$ vs $15 \%)$ at the significance level 41 of 0.05 and $80 \%$ statistical power based on a two-sided test.

42 Discussion: The results of this study will provide evidence for the efficacy and safety of 43 frozen cleavage embryo transfer compared with fresh cleavage embryo transfer in 44 women of advanced age undergoing IVF/ICSI. 
45 Trial registration: Chinese Clinical Trial Registry, ChiCTR2000029330. Registered on 25

46 Jan 2020.

47 Keywords: Frozen embryo transfer, advanced age, in vitro fertilization (IVF),

48 intracytoplasmic sperm injection (ICSI), live birth, randomized controlled trial.

49

50

51

52

53

54

55

56

57

58

59

60

61

62

63

64

65

66 
Previously, fresh embryo transfer was the common practice in most IVF centers,

69 whereas surplus embryos were frozen by a slow-freezing method and thawed for later

70 transfer when needed. The first case of live birth after frozen embryo transfer (FET) was

71 reported in 1984 . With the development of vitrification, the survival rates of thawed

72 embryos were greatly improved as compared with slow-freezing. Moreover, there are

73 some medical indications for elective FET such as genetic screening of embryos.

74 Observational studies suggest that FET seems to have better obstetric and perinatal

75 outcomes than fresh embryo transfer. Consequently, the clinical application of FET has

76 increased in recent years. In theory, FET under physiological conditions may improve the

77 chance of embryo implantation. Thus there were advocates proposing elective FET

78 strategy. However, high-quality evidence comparing the live birth rate after elective FET

79 versus fresh embryo transfer is limited. Recent meta-analyses of randomized controlled

80 trials (RCT) show that elective FET is associated with decreased risks of small for

81 gestational age, low birth weight and preterm birth but increased risks of large for

82 gestational age, high birth weight and pre-eclampsia. Of notice, previous RCT studies

83 were mainly conducted in young patients aged less than 35 years. It is unknown if

84 elective FET can improve the live birth rate in women of advanced age than fresh

85 embryo transfer. Therefore, we propose a RCT study to compare the cumulative live

86 birth rate of elective FET versus fresh embryo transfer in Chinese women of advanced

87 age. 
90 In the past 40 years, more than 7 million babies have been conceived by assisted

91 reproductive technologies (ART) treatment, mainly through in vitro fertilization (IVF) and

92 intracytoplasmic sperm injection (ICSI) (1). Increasing evidence suggests that ART

93 treatment is associated with adverse obstetric and perinatal outcomes, which may be

94 related to the genetic factors of patients, multiple pregnancies and ART procedures per

95 se $(1,2)$. To avoid multiple pregnancies, a single embryo transfer policy has been

96 implemented in many IVF centers. In Shanghai of China, this policy was compulsary for

97 all the women who are undergoing their first embryo transfer and no more than two

98 embryos are allowed to be implanted after the first transfer since January 2019.

99 However, even singletons conceived by ART are also at increased risk of adverse

100 pregnancy outcomes such as low birth weight, preterm birth, small for gestational age,

101 stillbirth, perinatal mortality and gestational diabetes compared with singletons

102 conceived spontaneously $(1,2)$.

103 Fresh embryo transfer is the common practice in most IVF centres, while surplus 104 embryos are cryopreserved and thawed for later transfer when needed. The first 105 successful pregnancy and the first case of live birth after frozen embryo transfer (FET) 106 was reported in 1983 and 1984 respectively $(3,4)$. In recent years, with the 107 development of vitrification, the survival rates of thawed embryos was greatly improved 108 as compared with slow-freezing (5). There are some medical indications for elective 109 frozen embryo transfer (eFET) or 'freeze-all' approach, including hydrosalpinx, increased 110 risk of ovarian hyperstimulation syndrome (OHSS), elevated progesterone levels, genetic 
111 screening, low responders, inadequate uterine cavity, high blood pressure and Zika virus

112 (6). Women undergo progestin-primed ovarian stimulation (PPOS) protocol or luteal

113 phase stimulation protocol also require eFET. Consequently, the clinical application of

114 FET has increased in recent years. For instance, the data from the USA showed that the

115 number of FET cycles increased by $82.5 \%$ from 2006 to 2012 , compared to a $3.1 \%$

116 increase in fresh embryo transfer cycles during the same period (7). Globally, the data

117 showed that the number of FET cycles increased by $27.6 \%$ from 2008 to 2010 (8).

118 A systematic review of eleven observational studies suggests that the transfer of

119 frozen thawed IVF embryos seem to have better obstetric and perinatal outcomes than

120 the fresh embryo transfer (9). The mechanism may be related to the improvement of

121 endometrial receptivity and the synchronization of embryos and endometrium under

122 physiological estradio levels during the frozen transfer cycle. For example, a large

123 retrospective cohort study in the US included 4071 normal responder patients with live

124 singleton births and found supraphysiologic estradiol was an independent predictor of

125 low birth weight in singletons born after fresh embryo transfer cycles (10). Thus, there

126 were advocates proposing eFET strategy $(11,12)$.

127 Although FET has been widely used, there are few high-quality studies comparing

128 the live birth rate after eFET versus fresh embryo transfer. Shapiro et al. performed two

129 small randomized controlled trials (RCTs) and showed that the clinical pregnancy rate of

130 the elective frozen blastocyst transfer group increased by about $15 \%$ in high responders

131 and $30 \%$ in the normal responders respectively, compared with the fresh blastocyst

132 transfer group $(13,14)$. A large RCT study in 2016 showed that frozen cleavage embryo 
133 transfer resulted in a higher rate of live birth, a lower risk of the OHSS, and a higher risk

134 of preeclampsia after the first transfer compared with fresh cleavage embryo transfer in

135 patients with polycystic ovary syndrome (PCOS) (15). In contrast, two large RCT studies

136 in 2018 showed no significant difference in live birth rate after elective frozen or fresh

137 cleavage embryo transfer in ovulatory women $(16,17)$, but FET was associated with a

138 lower risk of the OHSS (17). A recent large RCT study found a higher singleton livebirth

139 rate but increased risk of pre-eclampsia in ovulatory women with good prognosis

140 undergoing frozen single blastocyst transfer versus fresh single blastocyst transfer (18).

141 Compared with fresh embryo transfer, recent meta-analyses show that frozen embryo

142 transfer is associated with decreased risks of small for gestational age, low birth weight

143 and preterm birth but increased risks of large for gestational age, high birth weight and

144 pre-eclampsia $(19,20)$. Noteably, previous studies were mainly conducted in young

145 patients aged less than 35 years. It is unknown if eFET can improve the live birth rate in

146 women of advanced age than fresh embryo transfer. Therefore, we propose a

147 randomized controlled clinical trial to compare the cumulative live birth rate of eFET

148 versus fresh embryo transfer in women of advanced age.

149 The results of this study will provide the data for clinicians and infertile couples

150 whether eFET is beneficial for women of advanced age.

151

\section{Methods/Design}

153 Study design and setting 
154 This study is a single centre, parallel, double-blind, superiority randomized controlled

155 clinical trial (1:1 treatment ratio). Participants will be recruited at Shanghai First

156 Maternity and Infant Hospital. This protocol has been written in accordance with the

157 Standard Protocol Items: Recommendations for Interventional Trials (SPIRIT). The trial

158 design is summarized in Figure 1, whereas the schedule of enrolment, interventions and

159 assessments during the study period is shown in table 1.

160

161 Inclusion criteria

162 - Women aged 35-44 years

163 - Women who have normal ovarian reserve (AFC $>6$ follicles, or $\mathrm{AMH} \geqslant 1.2 \mathrm{ng} / \mathrm{ml}$ )

164 - Infertile couples scheduled for their first IVF/ICSI cycle.

165 - Women who will receive either gonadotrophin-releasing hormone agonist protocol

166 or gonadotrophin-releasing hormone antagonist protocol as their $\mathrm{COH}$ treatment.

167 - Informed consent.

168

169 Exclusion criteria

170 - Women with congenital or secondary uterine abnormalities, such as uterine

171 malformations including single-horned uterus, septate uterus or double uterus,

172 adenomyosis, uterine submucosal fibroids, intrauterine adhesions

173 - Couples undergoing preimplantation genetic testing (PGT)

174 - Women with hydrosalpinx

175 - Women with recurrent miscarriage 
176 - Women with polycystic ovarian syndrome (PCOS)

177 - Women with endocrine or metabolic abnormalities (pituitary, adrenal, pancreas,

$178 \quad$ liver or kidney)

179 - Women undergoing blastocyst transfer

180 - Natural cycles or IVM cycles

181 - Sperm donation or egg donation cycles

\section{Recruitment}

184 Infertile couples who come to the outpatient clinic to receive IVF/ICSI will be screened 185 by trained clinical team who are very familiar with the eligible criteria. Eligible patients 186 will then be approached by a member of the research team and explained the trial 187 details before the start of IVF/ICSI treatment. Couples will be offered time for 188 consideration to decide to participate the trial. If the couple agrees to participate, they 189 will make an appointment to sign the consent form in their next visit of clinic. Couples 190 who refuse to participate will be treated according to the conventional protocols at the 191 centre. The decision to refuse or withdraw will not affect their conventional clinical 192 treatments and the relationship with clinical practitioners.

193 The recruitment in the study centre will start in March 2020 and continue until the 194 needed number of participants is included, anticipated until February 2022.

\section{Randomization and blinding}

197 Eligible women will be randomized to single frozen embryo transfer or single fresh 198 embryo transfer. Randomization and allocation of patients to study arms will be 
199 performed on the day of the oocyte retrieval if $4 \sim 15$ oocytes are retrieved. Permuted

200 block randomization is controlled by collaborative investigators who are not involved in

201 the consulting and treatment procedure. When there is an eligible participant to be

202 enrolled into the study, Investigators will login the trial system (REDCap) to get

203 allocation of patients according to a computer-generated randomization list in a 1:1

204 ratio, with a variable block size of 2,4 or 6.

205 This study will be blinded to embryologists and laboratory technicians until the 206 completion of statistical analysis of this study. However, participants, clinicians, 207 investigators and nurses who conduct follow-up will not be blinded.

\section{Interventions}

210 All participants will receive controlled ovarian hyperstimulation $(\mathrm{COH})$ treatment, which

211 is performed by standard routines at the study centre. The selection of protocol will be

212 done by physicians, who are blinded for group allocation. In the gonadotrophin-

213 releasing hormone antagonist ( $\mathrm{GnRH}$-ant) protocol, all participants will be injected

214 gonadotropin (Gonal-F or Puregon or HMG) daily from day 2 or day 3 of menstrual cycle.

215 When at least one follicle has reached a diameter of $12 \mathrm{~mm}$ or on day 6 of ovarian

216 stimulation, GnRH antagonist (Cetrotide or Ganirelix) $0.25 \mathrm{mg}$ daily will be administered

217 subcutaneously until the trigger day (include the trigger day). For long GnRH-a protocol,

218 pituitary down-regulation will be initiated 7-10 days before the menstrual cycle with

219 GnRH agonist (subcutaneous Triptorelin $0.1 \mathrm{mg} / \mathrm{d}$ or intramuscular Triptorelin 1.25-

$2201.88 \mathrm{mg}$ one-time). After 10-14 days or on day 2 of menstrual cycle, gonadotropin 221 treatment will start. 
222 For all the protocols, menstrual cycle of patient includes spontaneous menstrual cycle,

223 and irregular menstrual cycle by the use of oral contraceptives (OC) or progestins.

224 Before $\mathrm{COH}$ treatment, baseline pelvic ultrasound, as well as baseline serum hormones

225 such as follicle stimulating hormone (FSH), luteinizing hormone (LH), estradial (E2),

226 progesterone (P) and $\beta-\mathrm{hCG}$ ) will be measured. The starting dose of gonadotrophins is

227 150-300IU/day for the first 4 days based on the characteristics of each patient.

228 Transvaginal ultrasound scanning and hormonal measurement will be repeated every 2-

2293 days to monitor follicle growth. The subsequent dose of gonadotrophin will be

230 adjusted according to the individual response. After two or more follicles reach a

231 diameter $\geq 18 \mathrm{~mm}, 250 \mathrm{ug}$ of hCG will be once injected on trigger day. In women with

232 hyper-response ( $\geq 15$ follicles $\geq 12 \mathrm{~mm}$ ), $0.2 \mathrm{mg}$ Triptorelin or $4000 \mathrm{IU}$ of hCG will be 233 administered.

234 Oocyte retrieval is scheduled for $36 \mathrm{~h}( \pm 2)$ after hCG injection. Fresh ejaculate semen

235 samples will be obtained on the day of oocyte retrieval, and are prepared by swim-up

236 protocol according to routines (21). For couple who take IVF, the procedure has been

237 previously described (21). All the oocytes will be inseminated with $2-5 \times 10^{6}$ per oocyte

238 motile spermatozoa approximately 39-42h after hCG injection. Gametes are then co-

239 incubated overnight at $37{ }^{\circ} \mathrm{C}$ under $5 \% \mathrm{O}_{2}$ and $6 \% \mathrm{CO}_{2}$ in the conventional incubators.

240 Assessment of fertilization are carried out about 16-18h (day 1) after fertilization. Then

241 zygotes are left in conventional incubators for a further 48 hours. For couple who take

242 ICSI, the oocytes will be denuded by hyaluronidase before micromanipulation. Only the

243 mature, metaphase-II (MII) oocytes with an extruded first polar body are microinjected. 
244 The procedure of ICSI has been previously described (22). After injection, oocytes are 245 transferred to the standard culture dishes in conventional incubators. Assessments of 246 fertilization and embryo quality after ICSI for the conventional incubators group are 247 identical with IVF. The cleavage embryo quality for both groups will be observed at 48 248 (day 2) or 72 (day 3) hours after oocyte retrieval. The embryos are scored according to 249 the quality, numbers, size of the blastomeres and the amount of anucleate 250 fragmentation.

251 For participants receive fresh embryo transfer, embryo transfer will be performed on 252 Day 3 after oocyte retrieval under ultrasound guidance. Surplus embryos will be frozen 253 according to the routines at the study centre. Luteal support is administered in the form 254 of vaginal progesterone (Crinone) $90 \mathrm{mg} / \mathrm{d}$ until the confirmation of biochemical 255 pregnancy, and will be maintained to 10 weeks of gestation. The progesterone will be 256 used until the menses when the biochemical pregnancy is not observed. Oral 257 progesterone (20mg b.i.d.; Duphaston) will be offered for women who appear vaginal 258 bleeding in case.

259 For participants undergo frozen thawed embryo transfer, patients with irregular menses 260 will receive oral E2 valerate (Progynova) 4-6mg/d on 2-3 days of subsequent artificial 261 menstrual cycle (by the use of oral contraceptives (OC) or progestins) within 6 months 262 after oocyte aspiration. Oral progesterone will be added if the endometrial thickness is $263 \geq 8 \mathrm{~mm}$. Patients with regular menses will have ovulation monitoring by transvaginal 264 ultrasound from day 12 of menstrual cycle. Oral progesterone will be added on the day 265 of ovulation. Frozen-thawed embryos will be transferred on Day 3 after progesterone 
266 initiation. The transfer procedure will be the same as that used for the fresh embryo 267 transfer. Oral medications will be continued at an unchanged dose until the 268 confirmation of biochemical pregnancy, and will be maintained to 10 weeks of gestation, 269 and it will be used until the menses when the biochemical pregnancy is not observed.

271 Follow-up

272 Urine and blood hCG will be measured 14 days after embryo transfer, and positive 273 results indicate biochemical pregnancy. If the gestational sac is observed with 274 ultrasonography on 7 weeks after transfer, clinical pregnancy will be confirmed. 275 Ongoing pregnancy is defined by the presence of a gestational sac with fetal heartbeat 276 after 12 weeks of gestation.

277 For women who are confirmed as ongoing pregnancy, they will be required to notify 278 researchers of the time of delivery. In 2 weeks after delivery, the information of 279 pregnancy (pregnancy complications, and fetus information), delivery information 280 (gestational age, delivery mode, placenta abnormality and/or delivery complications), 281 infant information (such as sex, birth weight, birth defect) will be collected by 282 completing forms.

284 Outcome measures

285 The primary outcome will be cumulative live birth of the trial IVF/ICSI cycle within 12 286 months of randomization. Live birth will be defined as a delivery of one or more living 287 infants ( $\geq 22$ week's gestation or birth weight more than $500 \mathrm{~g}$ ). 
288 For the effectiveness of the treatment, we will record these secondary outcomes in 289 terms of effectiveness:

290 - Fertilisation: defined as number of zygotes with 2PN (per woman randomised and 291 per oocyte retrieved).

292 - Available embryo: defined as number of embryos $\geq 4$ cells and $\leq 30 \%$ fragmentation 293 on day 3 observation.

294 - Good quality embryo: defined as number of embryos with $\geq 6$ cells and $\leq 30 \%$ 295 fragmentation developed from 2PN embryos on day 3 observation.

296 - Biochemical pregnancy: defined as blood $\mathrm{hCG} \geq 10 \mathrm{U} / \mathrm{L}$ at 14 days after embryo 297 transfer.

298 - Implantation: defined as the number of gestational sacs observed per embryo 299 transferred.

300 - Clinical pregnancy: defined as one or more observed gestational sac or definitive 301 clinical signs of pregnancy under ultrasonography at 7 weeks after embryo transfer 302 (including clinically documented ectopic pregnancy).

- Multiple pregnancy: defined as a pregnancy with two or more gestational sacs or 304 positive heart beats at 7 weeks of gestation.

305 - Ongoing pregnancy: defined as the presence of a gestational sac and fetal heartbeat 306 after 12 weeks of gestation.

307 For the safety of the treatment, we will record the following treatment complications as 308 secondary outcomes: 
- Ovarian hyperstimulation syndrome (OHSS): defined as exaggerated systemic

310 response to ovarian stimulation characterized by a wide spectrum of clinical and

311 laboratory manifestations. It is classified as mild, moderate, or severe according to

312 the degree of abdominal distention, ovarian enlargement, and respiratory,

313 hemodynamic, and metabolic complications.

314 - Miscarriage: defined as the spontaneous loss of an intra-uterine pregnancy prior to 31522 completed weeks of gestational age.

- Ectopic pregnancy: defined as the implantation takes place outside the uterine cavity,

317 confirmed by sonography or laparoscopy.

318 We will also collect the following obstetric and perinatal complications:

319 - Gestational diabetes mellitus (GDM)

- Hypertensive disorders of pregnancy (comprising pregnancy induced hypertension

$321 \quad$ (PIH); pre-eclampsia and eclampsia)

- Antepartum haemorrhage, including placenta previa, placenta accreta and

$323 \quad$ unexplained

- Preterm birth: defined as birth of a fetus delivered after 22 and before 37 completed

325 weeks of gestational age in participants confirmed ongoing pregnancy.

- $\quad$ Birth weight, including low birth weight (defined as weight $<2500$ gm at birth), very

327 low birth weight (defined as $<1500 \mathrm{gm}$ at birth), high birth weight (defined as $>4000$

328 gm at birth) and very high birth weight (defined as $>4500 \mathrm{gm}$ at birth)

- Large for gestational age (defined as birth weight >90th centile for gestation, based 330 on standardized ethnicity based charts) and small for gestational age (defined as less 
than 10th centile for gestational age at delivery based on standardized ethnicity

332 based charts)

- Congenital anomaly (any congenital anomaly will be included)

- Perinatal mortality: defined as fetal or neonatal death occurring during late pregnancy (at 22 completed weeks of gestational age and later), during childbirth, or up to seven completed days after birth.

338 Data management and monitoring

339 The data collected for the trial will be a mixture of routinely clinical data (such as 340 demographic data, fertility history, ART records), which are verifiable from the medical

341 record and questionnaire data. All researchers and physicians are required to receive 342 training classes and pass the test. Each participant will be assigned an appropriate code 343 number that is consistent with the allocated intervention, which will appear on all 344 report forms to maintain confidentiality.

345 All data are collected at baseline and follow-up through a standard clinical electronic 346 data collection system (EDC). Initially, all of the researchers and physicians will be 347 required to keep accurate and verifiable source notes in the medical record relevant to 348 each participant's eligible criteria of this trial. After recruitment of eligible participants, 349 trained assessors will take charge of the data input: they can log on to a secure data 350 portal with the individual ID, and upload the data from medical record to eCRF with the 351 personal trail ID of each participant. When the trial is close-out, all participant- 
352 identifiable data, such as consent forms, screening and identification logs will be stored

353 in the investigator site files, accessible only to delegated members of the study team.

\section{Sample size calculation}

356 According to the data of our centre, live birth rate among women aged $\geq 35$ years in the 357 control arm were around $15.0 \%$. Based on other studies within fertility care as well as 358 the discussion by gynaecologist and epidemiologists, we assumed that the minimal 359 clinical important difference to make frozen embryo transfer preferable over fresh 360 embryo transfer would be $8.0 \%$. To demonstrate this difference with two-sided test,

$3615.0 \%$ alpha-error, $80 \%$ statistical power, and taking consideration of dropout as $10 \%$, the 362 lowest numbers of participants we need to enrol for the study are 840 . The ratio 363 between test and control group will be 1:1.

365 Statistical analysis

366 Baseline characteristics will be described by descriptive analysis, and the balance 367 between the two arms will be assessed. For continues variables, the normality test will 368 be estimated using frequency histograms and the Shapiro test initially. If the parameters 369 are normally distributed then they will be presented as mean with standard deviation 370 (SD). If the parameters are non-normally distributed, their medians and inter-quantile 371 ranges (IRQs) will be reported. For categorical variables, we will present the proportions 372 of the two arms. In addition, we will also report the numbers of recruitment, 373 participants lost to follow-up, protocols violation, and other relevant descriptive data. 
374 Data analysis of this trial will follow the intention-to-treat principle, which includes all

375 randomized women in the primary comparison between the two arms. Per-protocol

376 analysis may be conducted as a secondary analysis. The primary outcome, cumulative

377 live birth rate, will be compared between the two arms using Pearson's chi-square test

378 or Fisher's exact test for the purpose of unadjusted analysis. We will also compute

379 unadjusted risk ratio $(\mathrm{RR})$ and its $95 \%$ confidence interval $(95 \% \mathrm{Cl})$. In the event of

380 prominent imbalance of potential confounders between the two arms, we will perform

381 multivariable Poisson Regression or Log-Binomial model to compute adjusted RR and its

$38295 \% \mathrm{Cl}$. Secondary outcomes will be compared between the two arms using the similar

383 approach described for the primary outcome.

384 For missing values regarding baseline characteristics, we will first perform analysis by

385 excluding missing values, we will then perform multiple imputation to impute missing

386 values and conduct subsequent analysis to estimate the robustness of the findings. For

387 loss to follow-up and protocol violation, we will attempt sensitive analyses to explore

388 the effect of these factors on the trial findings.

389 Primary and secondary outcomes will be compared between the two arms within

390 subgroups of different $\mathrm{COH}$ protocols in which the effects on outcomes might be

391 modified. Due to the concern over multiplicity of sub-group analysis, we will place

392 limited importance on subgroup findings.

393 All tests will be two-tailed, and differences with $p$ value $<0.05$ will be considered 394 statistically significant. 


\section{Safety}

397 All observed or volunteered adverse events, regardless of treatment group or suspected 398 causal relationship to intervention, will be recorded and reported to an independent 399 Data and Safety Monitoring Board (DSMB).

400 The investigator will inform subjects and the reviewing accredited medical research 401 ethics committee if anything occurs, on the basis of which it appears that the 402 disadvantages of participation may be significantly greater than was foreseen in the 403 research proposal. The study will be suspended pending further review by the 404 accredited medical research ethics committee, unless suspension would jeopardize the 405 subjects' health. The investigator will take care that all subjects are kept informed.

\section{Interim analysis}

408 The DSMB will perform an interim analysis within 3 months after the first 365 409 randomised participants have completed embryo transfer. They will do so using the 410 endpoint ongoing pregnancy, as data on live birth will not be available. The interim 411 analysis will be conducted using a two-sided significant test with the Haybittle-

412 Petospending function and a Type I error rate of 5\% with stopping criteria of $P<0.001$ ( $Z$ 413 alpha = 3.29). The study could be stopped prematurely based on the advice of the DSMB.

\section{Discussion}

416 Age is an important factor that affects woman's fertility. Compared with younger 417 women, older women have low ovarian reserve and a sharp decline in fertility. 
418 Increased maternal age is also associated with higher risk of pregnancy loss, obstetric 419 and perinatal complications, and chromosomal abnormalities of infants. Generally, 420 women aged at 35 years or older are considered as women of advanced age. With the 421 development of society, the reproductive age is gradually delayed, and the number of 422 ART cycles in women of advanced age has significantly increased. Live birth rate of ART 423 cycles decreases with age, due to poor response to controlled ovarian stimulation, poor 424 oocyte and embryo quality, and increased pregnancy loss. Women of advanced age are 425 classified as poor responders or a population of poor prognosis in ART cycles $(23,24)$.

426 It is unclear that whether frozen embryo transfer can improve the live birth rate of 427 women with advanced age as high-quality evidence is lacked. A retrospective cohort 428 study analyzed 1455 fresh blastocyst transfer cycles and 1455 frozen blastocyst transfer 429 cycles from 12 reproductive centers in the US conducted from 2009 to 2015 . The data 430 showed the FET group had significantly higher ongoing pregnancy rate than the fresh 431 transfer group (52\% vs $45.3 \%$, odds ratio (OR) :1.31,95\% Cl:1.13-1.51). The sensitivity 432 analysis suggested that FET may have a beneficial effect with increasing maternal age, 433 independently of progesterone levels before transfer (25). The data from the American 434 Association for Assisted Reproductive showed that between 2006 and 2012, the live 435 birth rate of women over 41 years old was higher after FET compared with fresh embryo 436 transfer, whereas in women aged 35-40 years the live birth rate of frozen embryo 437 transfer increased gradually and was higher than that of fresh embryo transfer in 2012 438 (7). These data suggest that FET may improve the outcome of ART therapy in women of 
439 advanced age. Taken together, randomized controlled trials are needed to provide the

440 efficacy and safety of frozen embryo transfer in women of advanced age.

441 Strengths of this trial include its randomized, controlled design and relatively large

442 sample size, which should minimize bias and increase validity and reliability of data. The

443 results of this study will provide evidence for the efficacy and safety of single frozen

444 cleavage embryo transfer compared with single fresh cleavage embryo transfer in 445 women of advanced age undergoing IVF/ICSI.

\section{Abbreviations}

449 AFC: antral follicle count; AMH: Anti-Mullerian hormone; ART: assisted reproductive 450 technology; $\mathrm{Cl}$ : confidence interval; $\mathrm{COC}$ : cumulus oocyte complexes; $\mathrm{COH}$ : controlled 451 ovarian hyperstimulation; CRF: case report form; DSMB: Data Safety and Monitoring 452 Board; E2: estradiol; EDC: electronic data collection; FET: frozen embryo transfer; FSH: 453 follicle stimulating hormone; GDM: gestational diabetes mellitus; GnRH: gonadotrophin454 releasing hormone; hCG: human chorionic gonadotrophin; HMG: human menopausal 455 gonadotropin; IVF: in vitro fertilization; ICSI: intracytoplasmic sperm injection; ITT: 456 Intent-to-treat; LH: luteinizing hormone; IVM: in vitro maturation; OHSS: ovarian 457 hyperstimulation syndrome; OC: oral contraceptives; P: progesterone; PCOS: polycystic 458 ovarian syndrome; PGT: preimplantation genetic testing; PIH: pregnancy induced 459 hypertension; PN: pronuclei; RCT: randomized controlled trial; RR: risk ratio; SD: 460 standard deviation; SPIRIT: Recommendations for Interventional Trials. 
Declarations

\section{Ethics approval and consent to participate}

464 This trial was approved by the institutional ethical committee of Shanghai First 465 Maternity and Infant Hospital on 20 January 2020 (Reference No.: KS2009). All 466 participants in the trial will provide written informed consent. The study was registered 467 on Chinese Clinical Trial Registry on 25 January 2020 (ChiCTR2000029330, 468 http://www.chictr.org.cn/showproj.aspx?proj=48725) and will be conducted according 469 to the principles outlined in the Declaration of Helsinki and its amendments, in 470 accordance with the Medical Research Involving Human Subjects Act, and using Good 471 Clinical Practice.

473 Consent for publication

$474 \quad$ Not applicable.

Availability of data and materials

477 The datasets used and analysed during the current study are available from the public 478 access repository ResMan of Chinese Clinical Trial Registry within six months after 479 completion of the study. The principle investigator will publish the results of the study 480 as soon as appropriate.

\section{Competing interests}

483 The authors have no conflicts of interest to declare. 
The trial is supported by a grant from the Chinese Medical Association (18010030732).

The funding bodies had no rule in the study design, implementation, analysis, manuscript, preparation or decision to submit this article for publication.

\section{Authors' contributions}

Study concept and design: MC, W-TL and B.W.M. Acquisition of data: MC, YW, XH, W-QL,

CS and ZM. Analysis and interpretation of data: MC, YW, XH, W-TL, W-QL and B.W.M. important intellectual content: YW, XH, W-QL CS, ZM and XT. Statistical analysis: MC, YW, XH, W-TL, W-QL and B.W.M. Study supervision: B.W.M and XT.

\section{Acknowledgements}

$498 \quad$ Not applicable.

\section{References}

502 1. Berntsen S, Soderstrom-Anttila V, Wennerholm UB, Laivuori H, Loft A, Oldereid 503 NB, et al. The health of children conceived by ART: 'the chicken or the egg?'. Hum 504 Reprod Update. 2019;25(2):137-58.

5052 2. Chen M, Heilbronn LK. The health outcomes of human offspring conceived by 506 assisted reproductive technologies (ART). J Dev Orig Health Dis. 2017;8(4):388-402.

5073 3. Trounson A, Mohr L. Human pregnancy following cryopreservation, thawing and 508 transfer of an eight-cell embryo. Nature. 1983;305(5936):707-9.

509 4. Zeilmaker GH, Alberda AT, van Gent I, Rijkmans CM, Drogendijk AC. Two 510 pregnancies following transfer of intact frozen-thawed embryos. Fertil Steril. 511 1984;42(2):293-6.

512 5. Rienzi L, Gracia C, Maggiulli R, LaBarbera AR, Kaser DJ, Ubaldi FM, et al. 513 Oocyte, embryo and blastocyst cryopreservation in ART: systematic review and meta- 
analysis comparing slow-freezing versus vitrification to produce evidence for the development of global guidance. Hum Reprod Update. 2017;23(2):139-55.

6. Basile N, Garcia-Velasco JA. The state of "freeze-for-all" in human ARTs. J Assist Reprod Genet. 2016;33(12):1543-50.

7. Prevention. CfDCa. 2012 Assisted Reproductive Technology Success Rates: National Summary and Fertility Clinic Reports. Atlanta: US Department of Health and Human Services. 2014.

8. Dyer S, Chambers GM, de Mouzon J, Nygren KG, Zegers-Hochschild F, Mansour R, et al. International Committee for Monitoring Assisted Reproductive Technologies world report: Assisted Reproductive Technology 2008, 2009 and 2010. Hum Reprod. 2016;31(7):1588-609.

9. Maheshwari A, Pandey S, Shetty A, Hamilton M, Bhattacharya S. Obstetric and perinatal outcomes in singleton pregnancies resulting from the transfer of frozen thawed versus fresh embryos generated through in vitro fertilization treatment: a systematic review and meta-analysis. Fertil Steril. 2012;98(2):368-77 e1-9.

10. Pereira N, Elias RT, Christos PJ, Petrini AC, Hancock K, Lekovich JP, et al. Supraphysiologic estradiol is an independent predictor of low birth weight in full-term singletons born after fresh embryo transfer. Hum Reprod. 2017;32(7):1410-7.

11. Evans J, Hannan NJ, Edgell TA, Vollenhoven BJ, Lutjen PJ, Osianlis T, et al. Fresh versus frozen embryo transfer: backing clinical decisions with scientific and clinical evidence. Hum Reprod Update. 2014;20(6):808-21.

12. Weinerman R, Mainigi M. Why we should transfer frozen instead of fresh embryos: the translational rationale. Fertil Steril. 2014;102(1):10-8.

13. Shapiro BS, Daneshmand ST, Garner FC, Aguirre M, Hudson C, Thomas S. Evidence of impaired endometrial receptivity after ovarian stimulation for in vitro fertilization: a prospective randomized trial comparing fresh and frozen-thawed embryo transfers in high responders. Fertil Steril. 2011;96(2):516-8.

14. Shapiro BS, Daneshmand ST, Garner FC, Aguirre M, Hudson C, Thomas S. Evidence of impaired endometrial receptivity after ovarian stimulation for in vitro fertilization: a prospective randomized trial comparing fresh and frozen-thawed embryo transfer in normal responders. Fertil Steril. 2011;96(2):344-8.

15. Chen ZJ, Shi $Y$, Sun $Y$, Zhang B, Liang $X$, Cao $Y$, et al. Fresh versus Frozen Embryos for Infertility in the Polycystic Ovary Syndrome. $N$ Engl J Med. 2016;375(6):523-33.

16. Vuong LN, Dang VQ, Ho TM, Huynh BG, Ha DT, Pham TD, et al. IVF Transfer of Fresh or Frozen Embryos in Women without Polycystic Ovaries. N Engl J Med. 2018;378(2):137-47.

17. Shi $Y$, Sun $Y$, Hao C, Zhang H, Wei D, Zhang $Y$, et al. Transfer of Fresh versus Frozen Embryos in Ovulatory Women. N Engl J Med. 2018;378(2):126-36.

18. Wei D, Liu JY, Sun Y, Shi Y, Zhang B, Liu JQ, et al. Frozen versus fresh single blastocyst transfer in ovulatory women: a multicentre, randomised controlled trial. Lancet. 2019;393(10178):1310-8.

19. Sha T, Yin X, Cheng W, Massey IY. Pregnancy-related complications and perinatal outcomes resulting from transfer of cryopreserved versus fresh embryos in vitro fertilization: a meta-analysis. Fertil Steril. 2018;109(2):330-42.e9.

20. Maheshwari A, Pandey S, Amalraj Raja E, Shetty A, Hamilton M, Bhattacharya S. Is frozen embryo transfer better for mothers and babies? Can cumulative meta-analysis provide a definitive answer? Hum Reprod Update. 2018;24(1):35-58.

21. Chen ZQ, Wang Y, Ng EHY, Zhao M, Pan JP, Wu HX, et al. A randomized triple blind controlled trial comparing the live birth rate of IVF following brief incubation versus standard incubation of gametes. Hum Reprod. 2019;34(1):100-8. 
565 22. Guo Y, Liu W, Wang Y, Pan J, Liang S, Ruan J, et al. Polarization microscopy 566 imaging for the identification of unfertilized oocytes after short-term insemination. Fertil 567 Steril. 2017;108(1):78-83.

568 23. Ferraretti AP, La Marca A, Fauser BC, Tarlatzis B, Nargund G, Gianaroli L, et al. 569 ESHRE consensus on the definition of 'poor response' to ovarian stimulation for in vitro 570 fertilization: the Bologna criteria. Hum Reprod. 2011;26(7):1616-24.

571 24. Poseidon G, Alviggi C, Andersen CY, Buehler K, Conforti A, De Placido G, et al. 572 A new more detailed stratification of low responders to ovarian stimulation: from a poor 573 ovarian response to a low prognosis concept. Fertil Steril. 2016;105(6):1452-3.

574 25. Wang A, Santistevan A, Hunter Cohn K, Copperman A, Nulsen J, Miller BT, et al. 575 Freeze-only versus fresh embryo transfer in a multicenter matched cohort study: 576 contribution of progesterone and maternal age to success rates. Fertil Steril. $577 \quad 2017 ; 108(2): 254-61$ e4.

578 
Figures

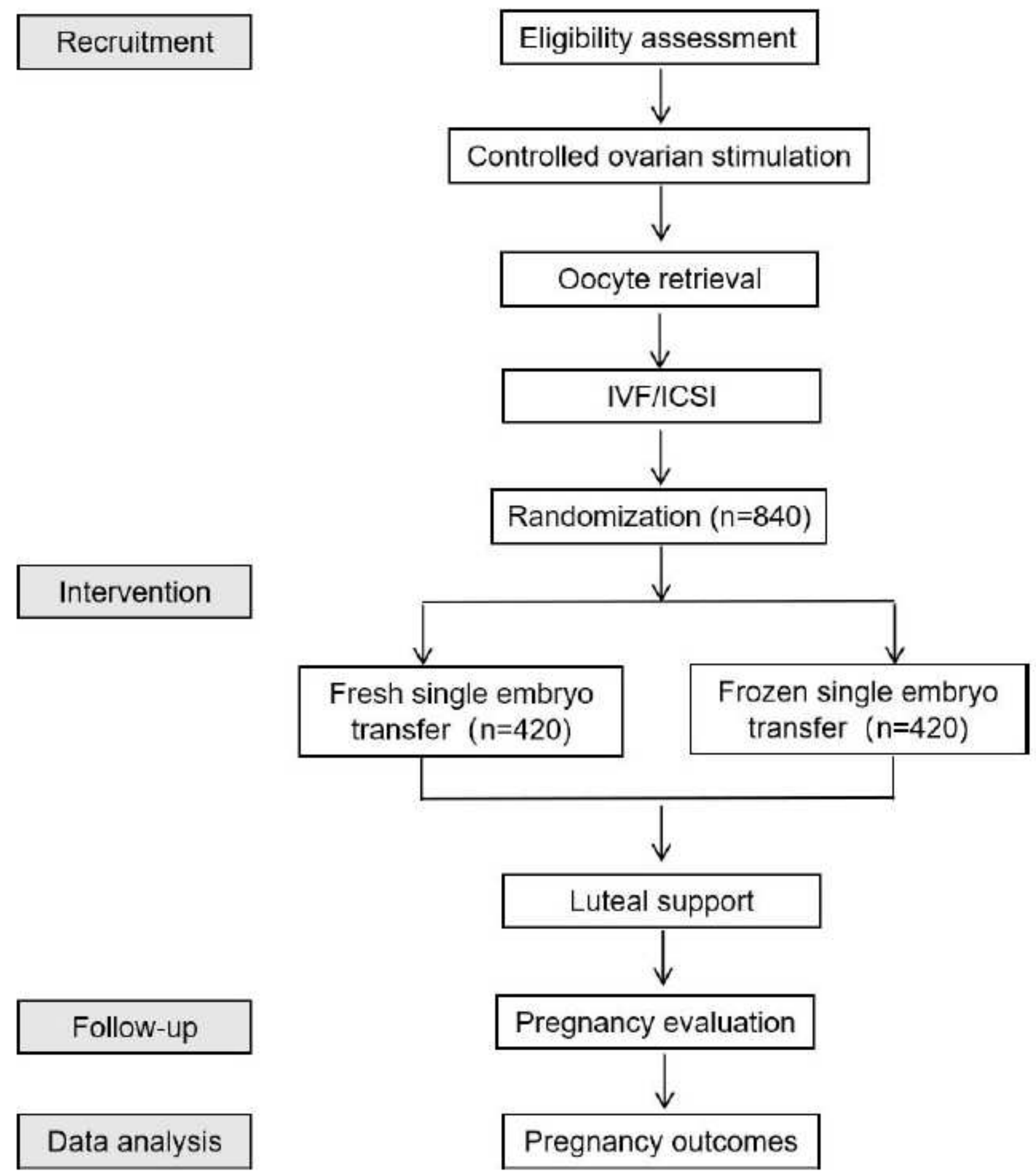

Figure 1

Flowchart followed the checklist of Standard Protocol Items: Recommendations for Interventional Trials (SPIRIT) showing patient enrolment, allocation, treatment and follow-up of participants. 


\section{Supplementary Files}

This is a list of supplementary files associated with this preprint. Click to download.

- Table1Scheduleofenrolment.pdf 